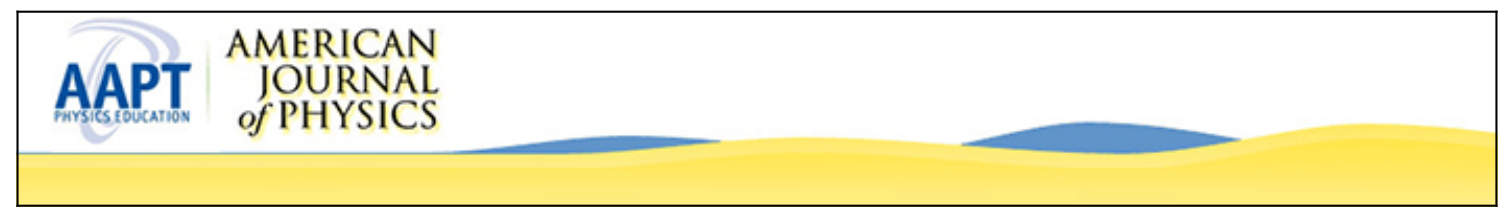

\title{
Experiment in Fourier Analysis
}

W. P. Lonc

Citation: American Journal of Physics 41, 401 (1973); doi: 10.1119/1.1987231

View online: http://dx.doi.org/10.1119/1.1987231

View Table of Contents: http://scitation.aip.org/content/aapt/journal/ajp/41/3?ver=pdfcov

Published by the American Association of Physics Teachers

\section{Articles you may be interested in}

Fourier-operator methods for parametric array analysis: Theory versus Experiment

J. Acoust. Soc. Am. 121, 3060 (2007); 10.1121/1.4781819

Multivariate Fourier and wavelet analysis of acoustic backscattering and environmental data from the acoustic surface reverberation experiment

J. Acoust. Soc. Am. 101, 3196 (1997); 10.1121/1.419339

Fourier Analysis

J. Acoust. Soc. Am. 86, 852 (1989); 10.1121/1.398173

Experiment on impulsive excitation, resonance, and Fourier analysis of a harmonic oscillator Am. J. Phys. 49, 31 (1981); 10.1119/1.12624

An Introduction to Fourier Analysis

Am. J. Phys. 30, 660 (1962); 10.1119/1.1942161

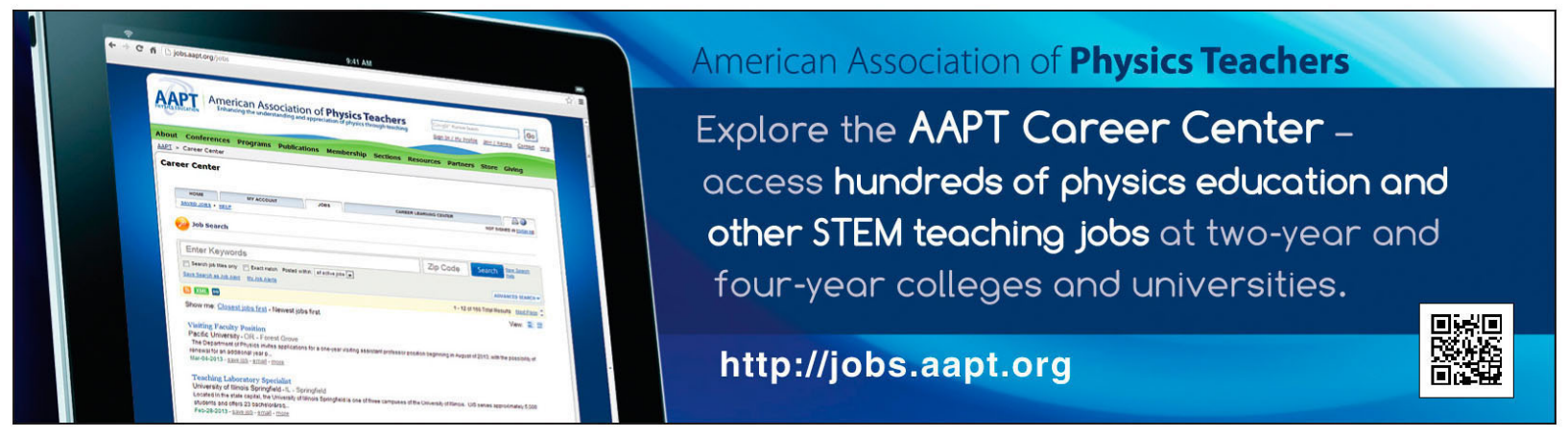




\section{Experiment in Fourier Analysis}

\author{
W. P. LONC, S.J. \\ Saint Mary's University \\ Halifax, Canada \\ (Received 8 June 1972; revised 28 August 1972)
}

Utilizing only commonly available components and instruments, construction and performance details are given for a system capable of extracting the sinusoidal components of a $10 \mathrm{kHz}$ square wave and a $10 \mathrm{kHz}$ halfwave rectified sine wave to at least the seventh harmonic with an amplitude accuracy of at least $10 \%$.

\section{INTRODUCTION}

In the study of wave phenomena at the undergraduate level, it seems justifiable to perform an experiment that verifies the existence of Fourier components. Experimental schemes for this purpose have appeared in these pages in the recent past. ${ }^{1}$ The present scheme, however, offers a means of "tuning-in" the Fourier components in a natural way, and because of its simplicity, could be duplicated and used in the course of a normal $3 \mathrm{~h}$ laboratory period.

On the assumption that a typical laboratory has an oscilloscope with a sensitivity of at least 10 $\mathrm{mV} / \mathrm{cm}$, and that the peak value of a sinusoidal voltage can be measured to no better than a few percent on this instrument, the quest for Fourier components may be terminated at about the seventh harmonic. It is also assumed that the typical laboratory has a sine/square generator capable of about $5-\mathrm{V}$ peak in the $10-\mathrm{kHz}$ region, and that the square wave is symmetrical to within a few percent. On these assumptions, the experiment is intended to detect and measure only the magnitudes and frequencies of sinusoidal components of a periodic voltage to approximately the seventh harmonic. In particular, both a square wave and a half-wave rectified sine wave are considered in this experiment.

\section{THEORY}

The basic idea behind Fourier analysis could be stated thus: A sufficiently well-behaved periodic nonsinusoidal waveform can be analyzed into an algebraic sum of sinusoidal waveforms. It goes without saying that such a statement suffers because of its brevity, but it does at least give the student a preliminary concept with which to proceed with the experiment. More explicitly, if the sufficiently well-behaved periodic waveform is symbolized as $V(t)$, then a simple form of the theory is expressed in the equation ${ }^{2}$

$$
V(t)=\frac{1}{2} V_{0}+\sum_{n=1}^{\infty}\left(a_{n} \cos n \omega t+b_{n} \sin n \omega t\right)
$$

where the first term is a measure of the de component of $V(t)$ and the remaining terms give the sinudoidal terms. For a specific form of $V(t)$, the de component is calculated by the formula

$$
V_{0}=\pi^{-1} \int_{0}^{2 \pi} V(t) d \omega t,
$$

and the amplitudes of the sinusoidal components are calculated by the formulas

$$
a_{n}=\pi^{-1} \int_{0}^{2 \pi} V(t) \cos n \omega t d \omega t
$$

and

$$
b_{n}=\pi^{-1} \int_{0}^{2 \pi} V(t) \sin n \omega t d \omega t
$$

where $n$ is successively given integral values (i.e., $1,2,3 \cdots$ ) and $\omega$ is the angular frequency. The angular frequency is obtained by the relationship $\omega=2 \pi / T$, where $T$ is the period of $V(t)$. In practice, the calculations are terminated once the amplitude for some value of $n$ becomes insignificant compared with the amplitude of the 
first harmonic. The definition of "significant" would, of course, depend on the accuracy either desired or attainable in the experiment.

As an example of the manner in which the theory is applied, suppose that $V(t)$ represents a square wave with no de component and just beginning its positive excursion at $t=0$. The calculation is relatively easy, yielding

$$
\begin{aligned}
V(t)=\left(4 V_{m} / \pi\right)\{ & \sin \omega t+\frac{1}{3} \\
& \sin 3 \omega t+\frac{1}{5} \sin 5 \omega t \\
& \left.+\frac{1}{7} \sin 7 \omega t+\cdots\right\}
\end{aligned}
$$

where $V_{m}$ is the peak value of the square wave. If the coefficients are normalized with respect to the coefficient of the first harmonic (i.e., of $\sin \omega t$ ), then the expression would be

$$
\begin{aligned}
V(t)=\sin \omega t+0.33 \sin 3 \omega t & +0.20 \sin 5 \omega t \\
& +0.14 \sin 7 \omega t+\cdots .
\end{aligned}
$$

For a half-wave rectified sine wave, Fourier analysis yields

$$
\begin{aligned}
V(t) & =\left(V_{m} / \pi\right)\left\{1+\frac{1}{2} \pi \sin \omega t-\frac{2}{3} \cos 2 \omega t\right. \\
& -(2 / 15) \cos 4 \omega t-(2 / 35) \cos 6 \omega t+\cdots\}
\end{aligned}
$$

where $V_{m}$ is the peak value of the half-cycle sine wave. Again, if the coefficients are normalized with respect to the fundamental, and the de term omitted, the expression would be

$$
\begin{array}{r}
V(t)=\sin \omega t-0.425 \cos 2 \omega t-0.085 \cos 4 \omega t \\
-0.037 \cos 6 \omega t+\cdots .
\end{array}
$$

Since $\cos \theta=\sin \left(\frac{1}{2} \pi-\theta\right)$ from elementary trigonometry, then each cosine term in the Fourier series may be expressed as $\sin \left(\frac{1}{2} \pi-n \omega t\right)$. Further, $\sin (-\theta)=-\sin \theta$, so that we could write $\sin \left(\frac{1}{2} \pi-n \omega t\right)=-\sin \left(n \omega t-\frac{1}{2} \pi\right)$. Hence, the term $-0.425 \cos 2 \omega t$ may be written as $0.425 \sin \left(2 \omega t-\frac{1}{2} \pi\right)$, and similarly for the remaining terms. Since the Fourier analyzer is not sensitive to phase, then there is no experimental difference between $\sin \left(n \omega t-\frac{1}{2} \pi\right)$ and $\sin (n \omega t)$. Hence, only the magnitudes and frequencies are the object of experimental verification, in terms of sine functions only.

\section{EXPERIMENTAL PROCEDURE}

\section{A. Preliminary Steps}

To demonstrate in a qualitative way the existence of Fourier components, say for a square wave, the variable capacitors in the circuit of Fig. 1(a) are slowly adjusted from a maximum value to a minimum value, which means that the resonant frequency of the circuit is being adjusted from some minimum to some maximum value. In the course of this adjustment, the oscilloscope screen will display sinusoidal waveforms for several well-defined values of the capacitance, and these sinusoidal waveforms will be of increasing angular frequency (i.e., of decreasing period $T$ ) as the capacitance is being adjusted towards its minimum value. Inasmuch as the "input" waveform is a periodic square wave, and the "output" waveforms are sinusoidal, then the existence of Fourier components is thereby demonstrated. At this point, it is instructive to switch the generator from square wave to sine wave (leaving the frequency unchanged) and to note that there is only one Fourier component.

The next step is to obtain quantitative data concerning amplitude and frequency of the components, but if the Fourier analyzer is used just as it appears in Fig. 1(a), the results will be quite disappointing, inasmuch as the relative magnitudes of the harmonics will bear little or no
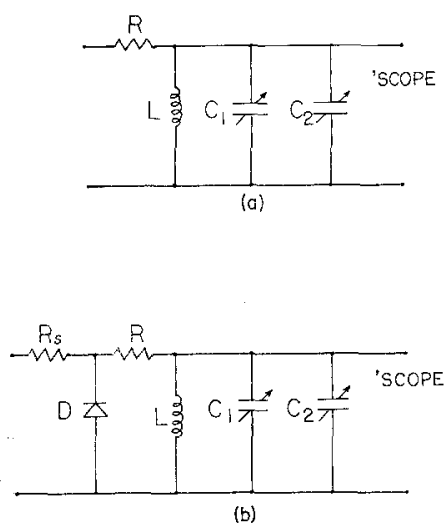

FIG. 1. Circuit details. (a) The basic Fourier analyzer. $L$ has a nominal value of $20 \mathrm{mH} ; C_{1}$ is a three-gang variable air capacitor connected in parallel to give approximately $1000 \mathrm{pF}$ maximum; $C_{2}$ is a decade capacitor (e.g., Heathkit $1 \mathrm{~N}-21$ ) with $1000 \mathrm{pF}$ minimum steps; $R=0.5 \mathrm{M} \Omega, \frac{1}{2} \mathrm{~W}$. (b) Clipper section added to the basic analyzer. $R_{s}=$ $20 \mathrm{k} \Omega, \frac{1}{2} \mathrm{~W} ; D$ can be practically any available diode. 
relationship to the calculated values. The problem lies in the frequency-dependent impedance of both the input circuit of the oscilloscope and of the analyzer circuit itself. Since these impedances are a relatively formidable quantity to predict for a typical student in sophomore or junior year, and since the objective of the experiment is to measure Fourier components in the course of a $3 \mathrm{~h} \mathrm{lab-}$ oratory period, it turns out that a calibration of the complete detection system by means of sine waves is the most convenient procedure.

With reference to Fig. 1(a), the calibration is performed as follows: The sine/square generator is switched to sine position, the frequency dial set to the fundamental frequency (e.g., $10 \mathrm{kHz}$ ), the resonant circuit tuned to this frequency, and the deflection on the oscilloscope noted (with the generator output at approximately $10 \mathrm{~V}$ peak to peak, the oscilloscope sensitivity control is set to give a convenient deflection). Without further adjustment of the oscilloscope sensitivity control, the capacitor of the analyzer is then adjusted so that the analyzer responds to the next harmonic (i.e., Fourier component) and the magnitude of the deflection on the oscilloscope is again noted. This procedure is repeated for each component originally observed at the beginning of the experiment. It is also assumed that the generator output voltage is being held constant throughout this procedure. For the circuit parameters used in our experiment, it was found that the system was about 10 times more sensitive at the seventh harmonic than at the fundamental.

Since the system sensitivity is frequency dependent, it is now necessary to construct a simple graph or table that displays a factor $A(n \omega)$ describing the response, defined by

$$
A(n \omega)=V(1 \omega) / V(n \omega),
$$

where $V(1 \omega)$ is the deflection on the oscilloscope when the analyzer is tuned to the fundamental, and $V(n \omega)$ is the deflection on the oscilloscope when the analyzer is tuned to the $n$th component. If $A(1 \omega)$ is given the value of one at the fundamental, then the values of $A(n \omega)$ will probably be less than one for all the higher components.

When the Fourier analyzer is then applied to a nonsinusoidal waveform, and since the system has
TABLE I. Comparison of observed and calculated harmonic amplitudes. (a) Square-wave input function. (b) Clipped sine-wave input function.

(a) Harmonic amplitudes for a square wave

\begin{tabular}{lcccccc}
\hline$n$ & Obs $^{\mathrm{a}}$ & Calc & Obs $^{\mathrm{b}}$ & Calc & Obs $^{\mathrm{c}}$ & Calc \\
\hline 1 & $\mathbf{1 . 0 0}$ & 1.00 & 1.00 & 1.00 & 1.00 & 1.00 \\
3 & 0.36 & 0.33 & 0.33 & 0.33 & 0.33 & 0.33 \\
5 & 0.22 & 0.20 & 0.19 & 0.20 & 0.21 & 0.20 \\
7 & 0.15 & 0.14 & 0.14 & 0.14 & 0.16 & 0.14
\end{tabular}

(b) Harmonic amplitudes for a clipped sine wave

\begin{tabular}{ccccc}
\hline$n$ & Obs $^{\mathrm{a}}$ & Calc & Obs $^{\mathrm{b}}$ & Calc \\
\hline 1 & 1.00 & 1.00 & 1.00 & 1.00 \\
2 & 0.35 & 0.42 & 0.41 & 0.42 \\
4 & 0.07 & 0.085 & 0.07 & 0.085 \\
6 & 0.03 & 0.036 & 0.02 & 0.036 \\
\hline \hline
\end{tabular}

a H.P. 209A generator and Heathkit IO-12 oscilloseope.

${ }^{b}$ H.P. 209A generator and Tektronix 503 oscilloscope.

c Eico 371 generator and Tektronix 503 oscilloscope.

already been discovered to respond to various harmonics in some manner described by the factor $A(n \omega)$, it only remains to multiply the deflection of each observed harmonic of the nonsinusoidal waveform by the appropriate factor to thereby obtain the true amplitude of that harmonic.

Once the circuit is connected, it is rather important to avoid moving the connecting wires, especially the ones from the $L C$ circuit to the oscilloscope, as this would constitute a change in impedance and consequently a change in system sensitivity.

\section{B. Square-Wave Analysis}

Assuming that the system has been calibrated as outlined above and the values of $A(n \omega)$ obtained, the circuit is then ready for use. If the generator is switched to the square-wave position, and the frequency set at $10 \mathrm{kHz}$, and the capacitors adjusted to resonate the circuit at $10 \mathrm{kHz}$, there should be a sine wave on the oscilloscope. The variable air capacitor is used to maximize the deflection on the oscilloscope, and then the magnitude of this deflection is recorded, and then later 
(c)

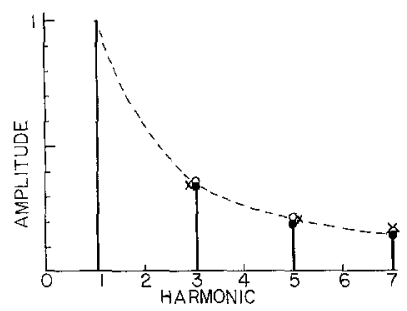

(b)

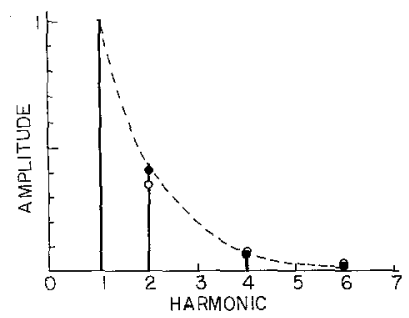

Fig. 2. Fourier spectra. The solid vertical lines represent the calculated values at each harmonic. The broken line is given to indicate more clearly the calculated amplitudes. By definition, the calculated and measured values for the first harmonic will be exactly equal in all cases. Spectra are for (a) square wave and (b) clipped sine wave.

multiplied by the appropriate factor $A(1 \omega)$. According to the theory, there should be no component at the second harmonic. However, there will probably be some kind of signal, but inasmuch as it is not sinusoidal, and on the plea that our system selectivity is not ideal, then this particular signal is disregarded. At the third harmonic, the signal is again nicely sinusoidal, and its deflection is again maximized by the air capacitor, its magnitude recorded, and then multiplied by $A(3 \omega)$ to obtain the true amplitude of this particular component. It will be found that the fifth and seventh harmonics are not as sinusoidal as the lower harmonies, but for the purposes of this experiment, they are sufficiently good. Results from only one run are given in Table I (a) for three different instrument combinations. It may be concluded that almost any available combination of instruments would give persuasive results.

\section{Clipped Sine-Wave Analysis}

A clipped sine wave may be obtained readily by simply incorporating almost any available diode as in Fig. 1(b). The waveform across the diode should be displayed on the oscilloscope to insure that the on and off times are equal to within $10 \%$ or less. Results from one run in each of two different instrument combinations are shown in Table $\mathrm{I}(\mathrm{b})$.

The experimental data may then be displayed in the form of a spectral diagram as in Fig. 2 for comparison with diagrams usually found in textbooks dealing with Fourier analysis.

\section{CONCLUSION}

With reference to the objectives and assumptions in this experiment, and taking into account the relative simplicity of the experiment, the Fourier components of a square wave can indeed be observed with an accuracy of $10 \%$ or better, and the components of a clipped sine wave with a somewhat lower accuracy. It is found that even economy-quality apparatus yields persuasive results.

1 F. H. Mitchell, R. N. Whitehurst, and Earl LeCroy, Amer. J. Phys. 28, 405 (1960) ; Seymour S. Alpert, Amer. J. Phys. 36, 606 (1968).

2 J. A. Edminister, Electric Circuits (McGraw-Hill, New York, 1965), Chap. 15. 